\title{
Resourceful Residual Energy Consumption in TDMA Scheduling for IoT-based Wireless Sensor Network
}

\author{
Amir Rizaan Rahiman*, Md. Ashikul Islam, Md. Noor Derahman
}

Department of Communication Technology and Network, Faculty of Computer Science and Information Technology, Universiti Putra Malaysia, 43400, Malaysia

\begin{tabular}{l} 
A R T I C L E I N F O \\
\hline Article history: \\
Received: 04 March, 2019 \\
Accepted: 23 April, 2019 \\
Online: 09 May, 2019 \\
\hline Keywords: \\
IoT \\
Wireless Sensor Network (WSN) \\
Time Division Multiplexing \\
Algorithm (TDMA) \\
Sensor node \\
Energy consumption \\
Broadcasting
\end{tabular}

\section{Introduction}

The recent revolution in the current Internet technology is the Internet of Things (IoT). It becomes a new communication technology enabling the development of the connection between different types of platforms like smart home, smart traffic monitoring system, autonomous vehicles, military operation, environment monitoring, sizeable agricultural area monitoring, cave and mining hole etc. [1]. Moreover, it becomes the main reason for the interconnection and co-operation between cloud computing, mobile Internet, distributed network and many more. Today, it slowly overtaking the modern wireless telecommunications, it's a novel paradigm. The Wireless Sensor Network (WSN) is the medium between this interconnection communication. It consists of sensor nodes handling the information transfer between source and sink or base station. Fig. 1 shows the common WSN architecture.

The usage amount of the WSN nodes growth as much as the IoT grows. All real-time systems totally depend on a wireless

\footnotetext{
*Amir Rizaan Rahiman, Email: amir_r@upm.edu.my
}

\begin{abstract}
A B S T R A C T
Recently, wireless sensor network (WSN) gets more concern due to the robustness in the echnologies includes smart home, smart farming, smart traffic control etc. wild range area. Routing distance, signal interference and routing computational cost give a significant impact to the WSN nodes lifetime. Unsynchronized node time allocation slot neighbor discovery are the main factors in the energy consumption issue faced by the performance. This paper discusses the optimization of distributed time division multiplexing algorithm (TDMA) slot scheduling for high-speed data link capacity. The E-T factor is based on the influence of residual energy and topology on the time slot allocation. Both node residual energy and topology information have shown respectable impact on the TDMA node slot allocation. Moreover, the numbers of and energy utilization can be reduced in the algorithm. been evaluated based on the previous experiment parameters with new high-speed data link. The experimental results have shown a significant improvement in residual energy consumption for the proposed optimized TDMA slot allocation
\end{abstract}

sensor for various tasks, such as data collection, environmental monitoring or even decision making according to the event occurs. Recently, advanced technology makes communication devices smaller with highly integrated low power consumption. Sometimes those devices are including tiny microprocessors supplied by the tiny scale of energy [2]. The WSNs is a combination of advanced micro devices that are used in collecting the IoT data due to its real-time processing system and easy deployment capability. It is cost effective, low power consumption, easy to implement with a multifunctional wireless system that bridge between physical space and information space. One of the greatest advantages of the WSN is it can distribute over a large geographical area which can be even between two cities or countries or can be into the ocean to monitor events like earthquake, tsunami etc.

However, the main limitation of WSNs is limited energy resource and lifetime where the sensor node is powered by small batteries. In addition, it is hard to change or add a new battery or energy resource due to rugged environments. For example, in the military and dangerous experimental works like checking volcano 
eruption, war zone monitoring, and deep-ocean search and rescue are benefited from the WSN. The period of monitoring would be a few days, months or years. For a large and long period network, only battery power is a suitable and efficient solution to power to the nodes. Generally, in a wireless environment, low energy efficiency and high data rate network become a challenging topic since the sensor nodes usually battery driven. Thus, managing the limited power supply in a proper and efficient way becomes an important task for the assisted IoT WSN [3]. Other factors of WSN nodes breakdown are continuously changing of network and wireless signal interruption. Power breakdown becomes a new challenge of the WSN time slot allocation and optimization.

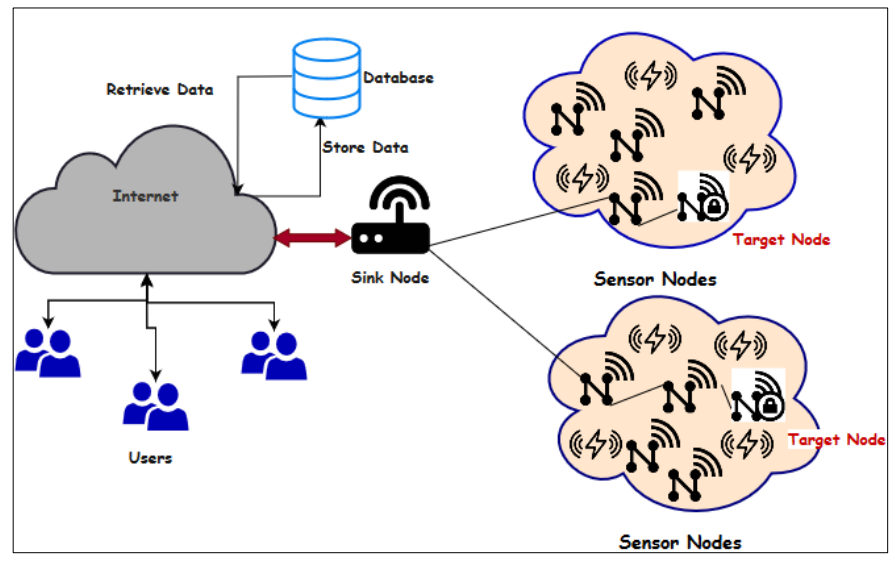

Figure 1: The WSN Architecture

Sensor nodes mobility and unfitting time slot allocation create a complicated network topology [4]. For instance, the dynamic network topology of the IoT networking and unutilized neighbor nodes discovery resulting in transmitting more message than usual. This will cause more energy consumption and reduce network performance. It is known that fast battery draining is among the major limitation of the WSN nodes. Therefore, most of the researchers are working to get better performance of the WSNs focusing on the time slot allocation topological [5 - 18]. However, these algorithms still have some limitations due to the node's distribution factor. Some of the solutions work efficiently for randomly distributed nodes but consumes more accessing time and energy in assigning the neighbor nodes. Another limitation occurs include limited data link capacity and network topology and coverage range. Recently, the network range (e.g., industrial and experimental) becomes broader (e.g., a manufacturing company, rural development monitoring, geographical network etc.). For example, even for smart traffic management system requires long distance network to monitor vehicle movement. On the other hand, every technology moving forward to catch up with high-speed Internets like $3 \mathrm{G}, 4 \mathrm{G}$ and some developed countries already tested the $5 \mathrm{G}$.

The two attentions of this paper are:

- To overcome the range limitation of the mesh topology by analyzing the optimized energy-topology (E-T-DRAND) with star topology that supports long-range network.

- To analyze the optimized algorithm behavior with a minimum of $10 \mathrm{Mbps}$ data link capacity. The reason is, the existing E-T-DRAND algorithm capable to allocate time slots without collision and can implement priority control with minimum message complexity that helps to reduce the energy consumption.

The simulation of this study was implemented on Object TCL (OTCL) environment in NS2.35 platform to evaluate the performance of optimized E-T-DRAND algorithm. The simulation results show improvements in message complexity, running time, number of rounds, and energy consumption. The rest of this paper is as follows. Background of the WSN scheduling algorithms and their related works are being discussed in Section 2. Section 3 explains the analysis mechanism of the proposed optimized algorithm. The performances of the experimental works are being discussed in Section 4. Section 5 concludes this paper.

\section{Related Works}

\subsection{The WSN}

The WSN consists of an outsized amount of sensor nodes where the nodes transfer the information from source to sink or base station. The sensor nodes play an important role to get better performance form the network, long service time efficient and low energy consumption. In addition, slot allocation, resources management makes the node energy consumption and performance getting better. The WSN main issue is establishing an energy-efficient routing protocol that gives a significant impact on the sensor lifetime. Several studies were developed to focus on improving the efficiency of the nodes energy utilization.

\subsection{DRAND and E-T-DRAND TDMA Slot Scheduling}

The TDMA energy-based distribution scheduling topology algorithm in IoT has been proposed by [5]. The mesh topology has been considered where the proposed E-T-DRANT (mainly analyzed the DRAND) being added an extra decision by referring to residual energy among the WSN nodes and the neighbor nodes. Since the time slot for both DRAND and E-T-DRAND is identical, the residual energy information with the priority control algorithm is being combined during allotting the time slot. The difference between DRAND and E-T-DRAND is the neighbor nodes discovery phase.

In E-T-DRAND, when one hop receives the broadcasted message with the energy information, the energy information table for the neighbor is being updated. Therefore, the two-hop neighbor receiving two-hop nodes of energy information. It is helpful for the nodes to manage nodes within two hops. The information of residual energy is being captured and stored in the information table for sending the requested time slot assignment. On the other hand, the time slot allocation control in E-T-DRAND scheduling is implemented by adopting an energy topology factor wherein the DRAND is done randomly. There are four stages for time slot allocation, IDLE, REQUEST, GRANT and RELEASE. The priority control algorithm helps in minimizing the node allocation process by putting some rules (e.g. less residual energy have higher priority). These rules ensure that only one node is applying for the time slot allocation to avoid a collision. The outcomes for both slots scheduling can be present by three complexity analysis, i) TDMA time slots allocation without collision, ii) Maximum and minimum message delays, and iii) Message transmission delay. 
The DRAND has been improved in terms of the balancing message complexity, time complexity. Therefore, the previous work improves the efficiency of the TDMA slot scheduling.

\subsection{Energy Efficiency in WSN Nodes}

Jian Shen et al. [6] proposed a protocol named energy-efficient centroid-based routing protocol (EECRP) for WSN-assisted IoT. The work proposed a clustering algorithm that constructs the node residual energy based on node position. The algorithm clusters the nodes to dead nodes and cluster head $(\mathrm{CH})$ nodes and has shown reducing the energy consumption average, especially for longrange communication without affecting the network lifetime. The Distributed TDMA Slot-scheduling (DTSS) algorithm for WSNs has been proposed by Bhatia and Hansdah [7]. The DTSS priority is to perform the scheduling with schedule length restriction requirement to a maximum degree of interference graph and accessing time. In the algorithm, the node needs to know only the intended receiver IDs and its advantage is the neighborhood nodes can take different slots simultaneously. To extend the wireless network lifetime in the IoT network, Hakan et al. [8] optimize the energy-aware for the Routing Protocol for Low Power and Lossy Networks (RPL). The work used Contiki OS which enables the emulation of extensive support for IoT protocols and being carried out by the Cooja simulation tool of Contiki OS which enables emulation of a wide variety of embedded hardware.

Nguyen et al. [9] work to focus on energy harvesting-aware routing for the WSN with the IoT. The algorithm is able to adapt with a different traffic load from the different application together with the residual energy and the arrival harvesting energy. The authors also proposed a model arrival-harvested energy prediction model at the WSNs nodes that include the stochastic characteristic of the ambient energy sources. In addition, a new parameter called "extra bakeoff" that combined with the energy prediction process to define the route to forward the packet with the cost metric has been introduced. In order to ensure the heuristic WSN service selection and composition with respect to the service classes, Zhou et al. [10] coincide sensor node with various sensing functionalities. The cooperative integration and strategy satisfy certain independent concurrent users request that control energy efficiency and minimize the WSN service problem. Compartmental clustering model of an opportunistic signal in WSN for nodes energy efficiency has been proved by [11]. The opportunistic signal of the optimal cluster partition is obtained through the compartmental model rather than the state-of-the-art attenuation models. Thus, the energy efficiency of optimal clustering is utilized from those signals which are carried out from various sources in an indoor environment.

Switching the roles between the sensor nodes to guarantee the WSNs energy consumption has been suggested by [12]. The nodes lifetime and fair evolution of energy level are guaranteed in this work by the positioning of sensor nodes such as a distance between the nodes in the network. Software-defined WSN has been proposed by [13] to reduce the data transmitting overhead between the sensor nodes. The proposed centralized routing algorithm increases the network lifetime in terms of the WSNs nodes consumption. Enabling IoT for underwater WSNs with balanced energy consumption by adaptive routing protocol for the sensor node been proposed by [14]. The underwater WSNs becomes difficult to work due to multifold limitations (e.g., power consumption, path loss, and inadequate bandwidth). To prolong the lifetime of underwater WSNs, a new routing protocol called balanced energy adaptive routing (BEAR) has been proposed.

Zhang et al. [15] proposed the energy-harvest wireless sensor network (EH-WSN) algorithm (environment-based harvesting energy) to prolong the network lifetime. However, energy harvesting poses lots of limitation which is difficult to achieve in the real-life. The algorithm adopts a double-stage capacitor structure to ensure node synchronization in situations without energy harvesting and uses an integrator to get ultra-low power measurement. Adjusting the sensor timeslot according to the environment changing to minimize the energy consumption in the TDMA channel accessing method has been proposed by [16]. In this work, all the sensor nodes used a fixed timetable slot for transmitting the data. The work is suitable for long time monitoring since the TDMA cannot be implemented in a scenario where the environment remains constant. If the environment is unchanged, the timeslot size remains the same, which is not the best solution for the WSN network.

A stochastic model in capturing the node predicted energy consumption by a schedulable sensor per cycle of operation has been proposed by [17]. The sensor node minimum and maximum energy consumption per cycle are being computed by the SemiMarkov theory can determine it expected life cycle. The particleswarm optimization solution being proposed by [18] to emphasize the optimal energy efficiency (EE) problem. The work optimizes the harvesting time and transmits power for non-orthogonal multiple access (NOMA)-based wireless powered WSN (WPSNs) with EE maximization for the wireless energy TDMA. A novel mode for clustering the cluster head $(\mathrm{CH})$ and the cluster formation in the WSN has proposed by [19]. The sensor network divides the nodes into zones (based on residual energy and distance) as per geographic locations. The clustering will be initiated when the $\mathrm{CH}$ residual energy become below the threshold value.

The above algorithms discuss and propose the distributed TDMA scheduling algorithms that improve the time slot allocation performance to some intensity by proposing several reliable solutions. Though, the difficulties, high energy consumption, message complexity and collision problem still ongoing which need more attention and further study.

\section{Optimized E-T-DRAND Time Slot Scheduling}

In this paper, the existing E-T-DRAND algorithm is being reevaluated and optimized by comparing with existing performance records from the aspect of message complexity, time complexity, number of experiment rounds, and energy utilization. The algorithm is being re-implemented in a randomly distributed network mode, where there is no central node and the algorithm being executed in more than one node at the same time. The neighbor nodes amount becomes the important factor and the node energy being represented as residual energy. The residual energy definition refers to the following "Energy Topology factor":

$$
E-T \rightarrow F(E i+\alpha x T i+N i)
$$

Parameter $E i$ refers to the residual energy of node $i$ while parameter $F$ is the sorting priority algorithm. The coefficient 
parameter $\alpha$ refers to the number not being allocated neighbor nodes and $\alpha \in[0,1]$. Ti is the number of neighbor nodes and $N i$ is the neighbor nodes energy information. The optimized algorithm improves the E-T-DRAND algorithm by adding neighbor node energy information among the WSN nodes where the characteristics are similar to the DRAND.

\subsection{Neighbor Node Discovery}

Fig. 2 shows the pseudo code for neighbor node discovery. The optimized algorithm uses the "HELLO" message containing the node residual energy information. In addition, the message also being used to manage the priority of the nodes time slot allocation. The information was used for updating energy information for the single-hop neighbor. For the two-hop neighbor, it is being discovered from the energy information table of the single-hop neighbor. Thus, when a start receiving the broadcast message, the node can get the energy information inward two hops. The node updates the released information with real-time energy information. During sending a request for time slot assignment, the nodes capture and store the residual energy information, thus, it helps the node to manage and optimize all the nodes within two hops from its own.

1. oneHopNeighbor[i]: Array of one hop neighbor nodes

2. twoHopNeighbor[j]: Array of two hop neighbor nodes

3. do \{ getOneHopNeighbor(i): collect data for one-hop neighbor

4. while (message.NdType $==$ FirstHop))

5. do $\{$ getTwoHopNeighbor(j): collect data for two-hop neighbor $\}$

6. while $\{$ getOneHopNeighbor $==$ true $\|$ message. NdType $==$ SecondHop $\}$ \} $\}$

Figure 2: Neighbor discovery pseudo code

\subsection{Implementation}

The most important entity in the WSN is proper TDMA slot allocation when the network dynamically changes. The improved E-T-DRAN is being implemented as the following:

- The energy topology factor always being scheduled together with the time slot allocation. During the time slot allocation request, at a first time, only one node can send a message. The time slot allocation process has four states namely, i) IDLE, if no request, ii) REQUEST, need to do time slot allocation or discover a new neighbor, iii) GRANT, the neighbor node time slot allocation successful and iv) RELEASE, no acknowledgement from the node.

- The priority control is being implemented together with time slot allocation. If any node has less residual energy, more neighbor node will get high priority. The first distributed priority order is based on the number of neighbor nodes and the "HELLO" message priority broadcast, see Fig. 3. More neighbor means node consume extra energy when the time slot allocation fails. On the contrary, when the node residual energy is lower, all next one-hop nodes neighbor will get higher priority, the additional overhead of these nodes increase energy consumption when the failure of the time slot allocation. If any node decides to allocate a time slot but the nodes in its two hops occupy less residual energy, the node will stop and declining its priority.

1. Int Node A;

2. Int Node B; \#one-hop neighbor

3. Int Node $\mathrm{C}$; \#two-hop neighbor

4. Ticket_number[]: Priority array

5. Value initialization: ticket_number[A] $+=$ neibourCount;

6. do $\{$ ticket_number $[\mathrm{B}]++\}$

7. while \{ has_unslotted_one-hop neibor_node B \&\& has_smaller_residual_energy(unslotted_one-hop neibor_node B\}

8. do $\{$ ticket_number $[\mathrm{C}]++$ \}

9. while \{ (has_unslotted_two-hop neibor_node C \&\& has_smaller_residual_energy(unslotted_two-hop neibor_node $\mathrm{C}\}$

10. do $\{$ Send a requested time slot packet, and transit current state to REQUEST;

11. while $\{\max ($ ticket_number[] $)==$ ticket_number[A] $)$

Figure 3: Priority control pseudo code

\section{Experimental Setup and Evaluation}

To evaluate the algorithm described in Section 3 is being implemented on the network simulator NS2.35 which is one of the best network simulators for multiple network models. The simulator version 2.35 has been chosen due to its scalable operating environment based on the Object TCL (OTCL) environment. To draw the nodes and the interface measurement NSG2.1.jar file is being used to draw the nodes and the interface measurement. The topology consists of 250 nodes, randomly being distributed on 300 X $300 \mathrm{~m}$ planes, see Fig. 4. The network nodes have $50 \mathrm{~m}$ broadcast communication range with a link capacity of $10 \mathrm{Mbps}$. The topology irregularity is extended to the maximum reflects by the randomness of the nodes. The experiment has been carried out 10 times similar to the configuration experiment in [5].

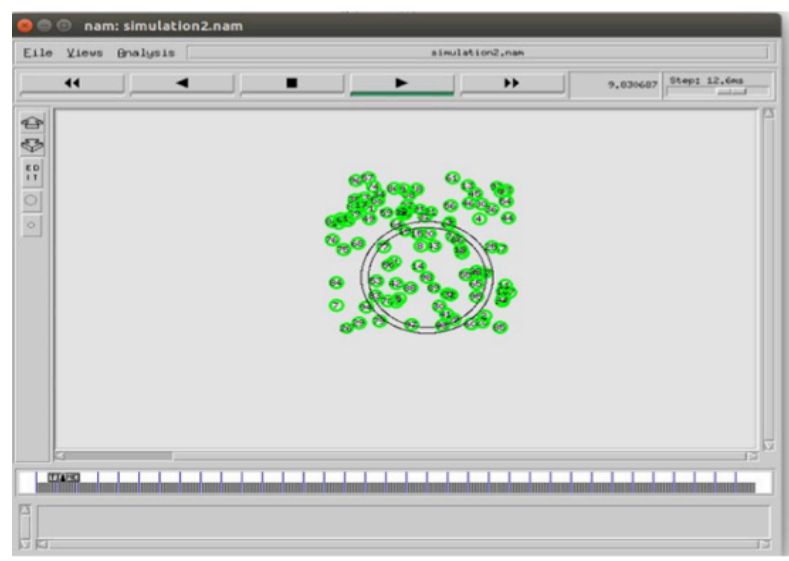

Figure 4: The topology structure of NS2.35 simulation.

The performance schemes of the experiment are evaluated and presented from different aspects that are message complexity, time complexity and energy consumption. The number of neighbor nodes varies from 5 to 55 in the two hops range and the simulation parameters are shown in Table 1 . We evaluate the proposed environment and algorithm by comparing with original ETDRAND algorithms from the aspects of message complexity, time 
complexity, number of rounds, energy consumption. As we know the distributed system does not have any central node so different nodes are executing the program and algorithms at a time. The performance evaluation of the experiment presents here through statistical results in figure 4.

Table 1: Performance Metrics

\begin{tabular}{|l|c|}
\hline Parameters & Values \\
\hline Number of nodes & 250 \\
\hline Link capacity & $10 \mathrm{Mbps}$ \\
\hline Broadcasting range & $50 \mathrm{~m}$ \\
\hline Initial energy & $1900 \mathrm{~mA}$ \\
\hline Receiving energy & $15 \mathrm{~mA}$ \\
\hline Transmitting energy & $20 \mathrm{~mA}$ \\
\hline Idle energy & $5 \mathrm{~mA}$ \\
\hline Average delay & $1 \mathrm{~s}$ \\
\hline Message Selection & Randomly \\
\hline
\end{tabular}

\subsection{Message Complexity}

For the experiment, the message selection is randomly and message complexity metric refers to a number of messages being broadcasted over any nodes. The number of messages broadcasted depending on the node neighbor size for the time slot allocation.

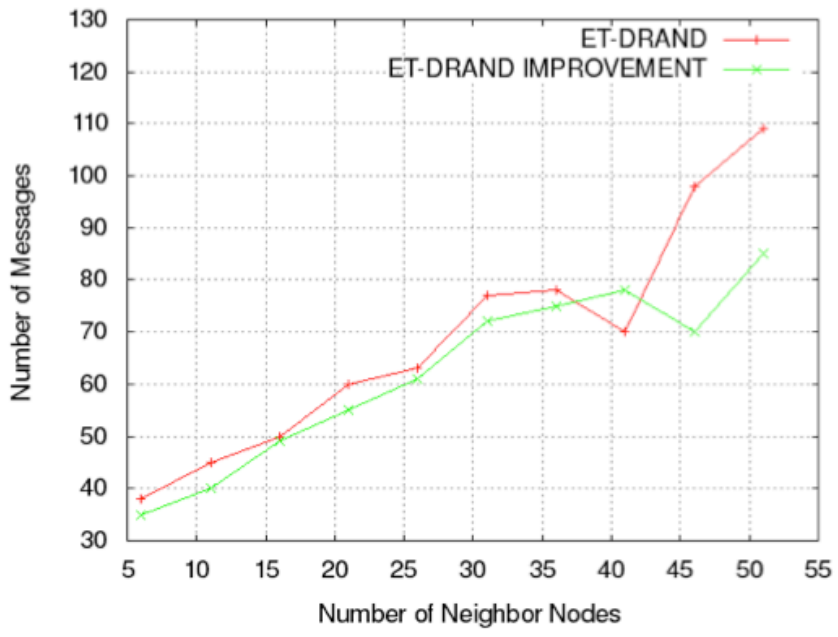

Figure 5: Number of messages in time slot allocation.

The average number of messages being broadcasted is shown in Fig. 5. The figure shows the average number of messages for a node to acquire a time slot in different size of neighbor nodes, where the performance between the existing result and the optimized algorithm is similar when the neighbor modes less than 40. When the number of the neighbor node increases more than 40 , then the existing experiment result takes more messages due to a low datal ink rate. The experiment result takes $20.7 \%$ less than the previous experiment because when the data link capacity has been increased. This result shows that with high data link rate, the optimized E-T-DRAND algorithm reduces message complexity.

\subsection{Running Time Complexity}

Fig. 6 presents the average running time of existing ETDRAND algorithm and the improvement result in the range of the different neighbour nodes successfully allocating the time. When the number of nodes reaches more than 30 , the running time keeps increase. As we know, more nodes mean many messages and many collisions. Therefore, when the number of nodes more than 30 , the collisions happened regularly due to collision slot request occurs continuously. However, the ET-DRAND algorithm performs better than DRAND algorithm when the number of nodes increases and the amount of message increase. The result confirms the optimized work takes almost the equal running time for time slot allocation and resource allocation with the previous algorithm.

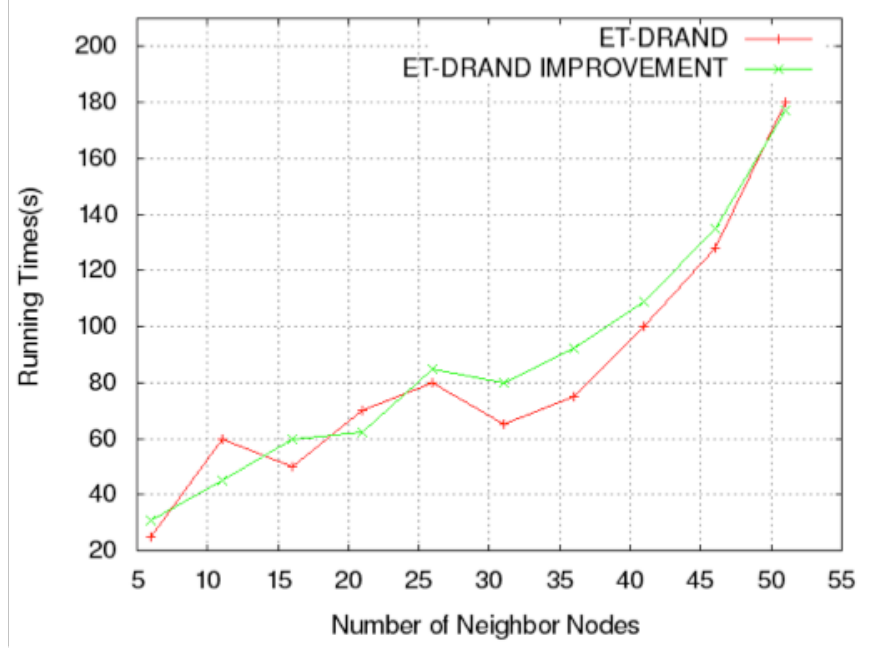

Figure 6: Average running time in time slot allocation.

\subsection{Number of Rounds}

Note that, this experiment is being carried out using a highspeed data link Internet. Fig. 7 above shows the average number of rounds successfully took to complete the time slot allocation. The running time shows the number of rounds/circle the experiment took to discover all the neighbor nodes. With the number of neighbour nodes increases, it is necessary to allocate time slots after multiple rounds.

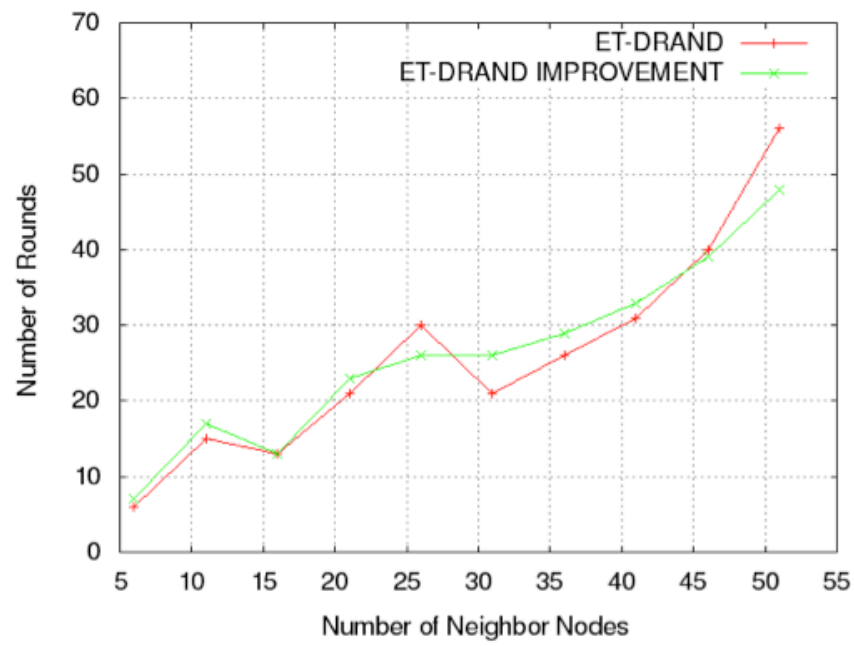

Figure 7: Number of rounds required in time slot allocation.

On the other hand, the running time, the collision increases with the increase of nodes. As mentioned above, the high data link shows an improvement when the neighbor nodes are above 45 . 
The experiment takes less round in allocating successful time slot and increase the resources allocation.

\subsection{Average Energy Consumption}

Fig. 8 shows the node average energy consumption for different size of neighbor. The experiment result shows less energy consumption when the neighbor nodes less than 20 . On the contrary, the result shows the similarity with the previous experiment. However, when there are more than 40 nodes the nodes consume 5-6\% more energy. This is due to the high collision. Since the experiment was done using high data link Internet, so more messages are passed, and more collisions occur. The experiment result proves that E-T-DRAND stills the best solutions in terms of time slot allocation and energy consumption.

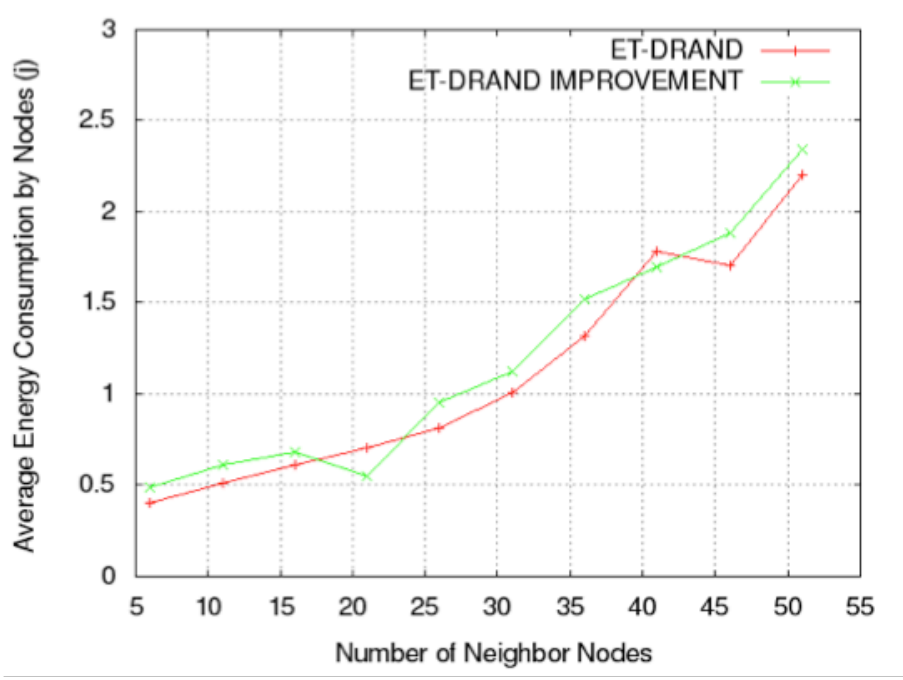

Figure 8: Neighbor nodes average energy consumption.

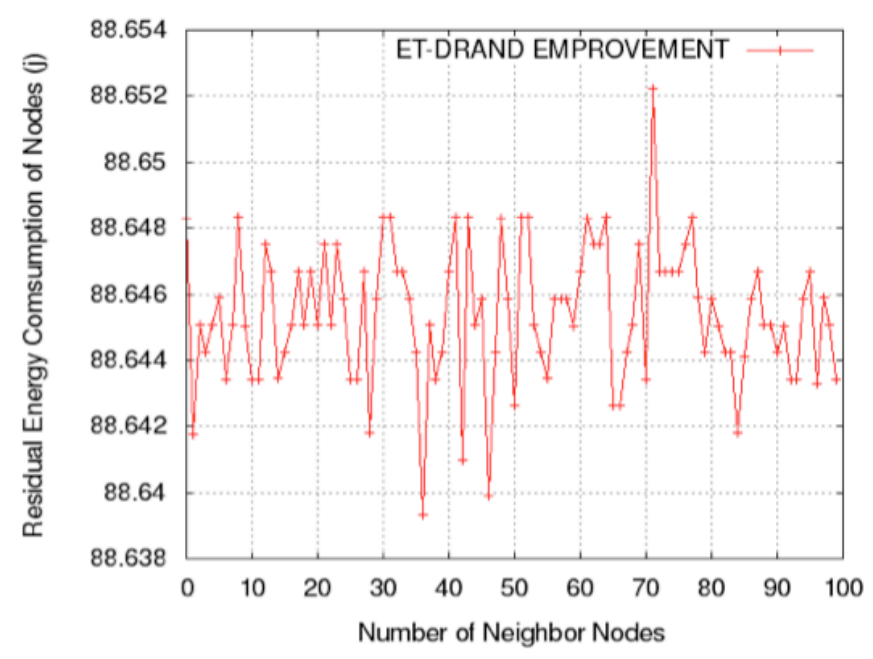

Figure 9: Neighbor nodes residual energy consumption.

\subsection{Neighbor nodes residual energy consumption}

Fig.9 summarizes the average neighbor nodes energy consumption after the experiment being repeated 10 times. It shows that the nodes group between 30 and 40 consumes less energy than others while the highest energy consumption hold by the neighbor nodes 70. This residual energy is to observe the node lifetime and nodes performance.

\section{Conclusion}

With the IoT, anything can be controlled and monitored, but for the WSN the power limitation and resources utilization issues make a boundary limit for the network performance improvement. In the distributed network environment, power consumption issues become the main challenges where lacking concentration on time and energy consumption of the WSN nodes makes IoT improvement slowly. In this study, the proper way for time slot allocation by combining with energy topology factor has been investigated. The allocation of the time slot for the MAC protocol in WSN have been analyzed and present the behavior of the algorithm in the mesh topology. The randomly distributed nodes causing the collision would increase the execution time. By updating the node time allocation with energy topology, the energy consumption of neighbor node can be reduced due to the randomness. The energy topology factor of the distributed TDMA slot-scheduling algorithm improves the percentage of nodes performance and reduces message complexity. The world moving for fast Internet like $4 \mathrm{G}$ and $5 \mathrm{G}$ where the $5 \mathrm{G}$ can be one of the best ways to improve communication. In future, implementing ET-DRAND algorithm with the $5 \mathrm{G}$ networks that is our next goal in reducing energy consumption and improves the WSN for the IoT to collect huge data. Since the big data made a huge impact on information technology and business, both the WSN and the IoT indirectly play their rules.

\section{Conflict of Interest}

The authors declare no conflict of interest.

\section{Acknowledgment}

The authors wish to thank the reviewers for their constructive comments and suggestions helped in improving this research paper and Universiti Putra Malaysia for providing the funding in carrying out this study.

\section{References}

[1] Y. Yang, M. Zhong, H. Yao, F. Yu, X. Fu, O. Postolache, "Internet of things for smart ports: technologies and challenges" IEEE Instru. Meas. Mag., 21(1), $34-43$, 2018. https://doi.org/10.1109/MIM.2018.8278808

[2] M. Gautam, V. Sejwar, "A Brief Review of WSN Energy and Routing Protocols" in International Conference on I-SMAC (IoT in Social, Mobile, Analytics and Cloud) (I-SMAC), Palladam, India, 849 - 855, 2017. https://doi.org/10.1109/I-SMAC.2017.8058299

[3] Z. Ning, Q. Song, Y. Yu, Y. Lv, X. Wang, X. Kong, "Energy-aware cooperative and distributed channel estimation schemes for wireless sensor networks" Int. J. Commun. Syst., 30(5), e3074, 2017. /doi/full/10.1002/dac.3074

[4] V. Gabale, B. Raman, P. Dutta, and S. Kalyanraman, "A classification framework for scheduling algorithms in wireless mesh networks" IEEE Commun. Surveys Tuts., 15(1), $199 \quad-\quad 222,2013$. https://doi.org/10.1109/SURV.2012.022412.00068

[5] Y. Li, X. Zhang, J. Zeng, Y. Wan, F. Ma, "A distributed TDMA scheduling algorithm based on energy-topology factor in internet of things" IEEE Access, 5, 10757 - 10768, 2017. https://doi.org/10.1109/ACCESS.2017.2710304

[6] J. Shen, A. Wang, C. Wang, P. C. Hung, C. Lai, "An efficient centroid-based routing protocol for energy management in WSN-assisted IoT" IEEE Access, 5, 18469 - 18479, 2017. https://doi.org/10.1109/ACCESS.2017.2749606

[7] A. Bhatia, R. C. Hansdah, "A Distributed TDMA Slot Scheduling Algorithm for Spatially Correlated Contention in WSNs" in $27^{\text {th }}$ International Conference on Advanced Information Networking and Applications Workshops (AINA), Barcelona, Spain, 377 - 384, 2013. https://doi.org/10.1109/WAINA.2013.23 
[8] H. Erdol, S. Gormus, M. C. Aydogdu, "A Novel Energy Aware Routing Function for Internet of Things Networks" in $10^{\text {th }}$ International Conference on Electrical and Electronics Engineering (ELECO), Bursa, Turkey, 1314 $1318,2017$.

[9] T. D. Nguyen, J. Y. Khan, D. T. Ngo, "An Effective Energy-HarvestingAware Routing Algorithm for WSN-based IoT Applications" in IEEE International Conference on Communications (ICC2017), Paris, France, 2017. https://doi.org/10.1109/ICC.2017.7996888

[10] Z. Zhou, J. Xu, Z. Zhang, F. Lei, W. Fang, "Energy-efficient optimization for concurrent compositions of WSN Services" IEEE Access, 5, 19994 - 20008, 2017. https://doi.org/10.1109/ACCESS.2017.2752756

[11] S. Kumar, "Compartmental modeling of opportunistic signals for energy efficient optimal clustering in WSN", IEEE Commun. Lett., 22(1), 173 - 176, 2018. https://doi.org/10.1109/LCOMM.2017.2763948

[12] S. Hamrioui, P. Lorenz, "ES-WSN: Energy Efficient by Switching between Roles of Nodes in WSNs" in 2015 IEEE Global Communications Conference (GLOBECOM), San Diego, CA, USA, 2015. https://doi.org/10.1109/GLOCOM.2015.7417047

[13] F. Junli, W. Yawen, S. Haibin, "An Improved Energy-Efficient Routing Algorithm in Software Define Wireless Sensor Network" in 2017 IEEE International Conference on Signal Processing, Communications and Computing (ICSPCC), Xiamen, China, 2017. https://doi.org/10.1109/ICSPCC.2017.8242610

[14] N. Javaid, S. Cheema, M. Akbar, N. Alrajeh, M. S. Alabed, N. Guizani, "Balanced energy consumption based adaptive routing for IoT enabling underwater WSNs" IEEE Access, 5, 10040 - 10051, 2017. https://doi.org/10.1109/ACCESS.2017.2706741

[15] Y. Zhang, H. Gao, S. Cheng, J. Li, "An efficient EH-WSN energy management mechanism" Tsinghua Science and Technology, 23(4), 406 418, 2018. https://doi.org/10.26599/TST.2018.9010034

[16] A. Biazi, C. Marcon, F. Shubeita, L. Poehls, T. Webber, F. Vargas, "A Dynamic TDMA-based Sleep Scheduling to Minimize WSN Energy Consumption" in 2016 IEEE 13th International Conference on Networking, Sensing, and Control (ICNSC), Mexico City, Mexico, 2016. https://doi.org/10.1109/ICNSC.2016.7478994

[17] V. Agarwal, R. A. Decarlo, L. H. Tsoukalas, "Modeling energy consumption and life time of a wireless sensor node operating on a contention-based MAC protocol” IEEE Sensor, 17(16), 5153 - 5168, 2017. https://doi.org/10.1109/JSEN.2017.2722462

[18] M. Song, M. Zheng, "Energy efficiency optimization for wireless powered sensor networks with nonorthogonal multiple access" IEEE Sensors Letters, 2(1), 1 - 4, 2018. https://doi.org/10.1109/LSENS.2018.2792454

[19] A. Chunawale, S. Sirsikar, "Minimization of Average Energy Consumption to Prolong Life Time of Wireless Sensor Network" in 2014 IEEE Global Conference on Wireless Computing \& Networking (GCWCN), Lonavala, India, 2014. https://doi.org/10.1109/GCWCN.2014.7030887 